Letter to Editor

\title{
Cardiovascular disease is common among patients with systemic lupus erythematosus
}

Volume 5 Issue 3 - 2016

\author{
Fathia Ehmouda Zaid,' Remi Abudelnbi² \\ 'Department of Rheumatology, Benghazi University, Libya \\ ${ }^{2}$ Department of Medicine, Benghazi University, Libya
}

Correspondence: Fathia Ehmouda Zaid,Assistant professor, Department of Rheumatology in Faculty of Medicine, Benghazi University, Libya, Tel 219000000000,

Email fatehmoda@yahoo.com

Received: June 23, 2016 | Published: July II, 2016
Patients with Systemic Lupus Erythematosus have increased morbidity and mortality from cardiovascular disease. Morbidity includes accelerated, premature atherosclerosis and valvular heart disease..$^{2-3}$

A variety of cardiac manifestations can be seen in Systemic Lupus Erythematosus, The most common pericarditis, Pericardial effusions may be asymptomatic. Myocardial involvement is rare $(<5 \%$ of patients) and typically occurs in the presence of generalized Systemic Lupus Erythematosus activity. Cardiac MRI has been used to detect clinical and subclinical myocardial involvement in Systemic Lupus Erythematosus. Unlike many other manifestations of Systemic Lupus Erythematosus, the valvular lesions in Systemic Lupus Erythematosus may occur at any time, and their presence does not correlate with disease activity.

Symptoms and severity of the cardiac disease in Systemic Lupus Erythematosus are linked to the pattern of auto antibodies expressed, variable expression of antibodies to ds-DNA, Ro or anticardiolipin. ${ }^{4}$ The risk of coronary heart disease is greatly increased in Systemic Lupus Erythematosus patients compared with an age and sexmatched segment of the general population. Patients with Systemic Lupus Erythematosus have an increased prevalence of traditional risk factors, and the disease itself and treatment with glucocorticoids are powerful risk factors for coronary heart disease. ${ }^{5}$

The variable diagnostic of cardiac disease from the history and physical examination, immunology, imaging, andhistology. Echocardiography is an effective, non-invasive method of documenting the presence of cardiac diseases, transthoracic echocardiography is more than acceptable in routine practice, Transesophageal echocardiography may be needed and is thus more sensitive. ${ }^{6}$ Other imaging has been used Screen of Coronary artery diseases in asymptomatic patients with SLE such as myocardial perfusion scanning; cardiac CT angiography, and stress echocardiography and cardiac MRI .become more widespread in clinical practice replace invasive angiography. ${ }^{7-8}$

End myocardial biopsy is definitive histological diagnosis and potentially low is still widely recommended..$^{9}$ Our study was to estimate the cardiovascular disease manifestation of systemic lupus erythematosus, correlation with disease activity, morbidity and mortality.

Cardiovascular disease was observed in $47 \%$ our patients with systemic lupus erythematosus, myocarditis and pulmonary hypertension are most frequent and significant correlation with disease activity compared with pericardial and valvular heart disease, which may be related to lack screen asymptomatic patients with systemic lupus erythematosus (SLE) and the use of less sensitive echocardiographic technique transthoracic echocardiography. ${ }^{6}$

We demonstrated (Table 1) a significant correlation between cardiovascular disease in systemic lupus erythematosus with disease activity use SLEDAI, pulmonary hypertension (PAH) and myocarditis. No significant difference in pericarditis, pericardial effusion and coronary artery disease. Which presence in asymptomatic patients with systemic lupus erythematosus or no had disease activity of systemic lupus erythematosus.

Table I Correlation between cardiovascular disease (CVD) and disease activity, specific organ involvement, morbidity and mortality

\begin{tabular}{|c|c|c|}
\hline \multicolumn{3}{|c|}{ Cardiac Manifestation of Systemic Lupus Erythematosus 32(47.1\%) } \\
\hline Disease Activity SLEDAI & & $(r=476 * p=0.000)$ \\
\hline Pulmonary Hypertension & $12(17.6 \%)$ & $\left(r=.257^{*}, p=.035\right)$ \\
\hline Pericarditis & $8[11.8 \%]$ & $(r=.210, p=.086)$ \\
\hline Pericardial Effusion & $6[8.8 \%]$ & $(r=0.079, p=.520)$ \\
\hline Myocarditis & $4[5.9 \%]$ & $\left(r=272^{*}, p=.027\right)$ \\
\hline Valvular Lesions (Endocarditis) & I[1.5\%] & $(r=.112, p=.362)$ \\
\hline Coronary Artery Disease & $\mathrm{I}[1.5 \%]$ & $(r=.075, p=.544)$ \\
\hline
\end{tabular}


Table Continued...

\begin{tabular}{ll}
\hline Correlation Specific Organ Involvement, Morbidity And Mortality \\
\hline Mucocutaneous & $(r=.091 p=.459)$ \\
Musculoskeletal (MSK) & $(r=.110 p=.373)$ \\
Renal Disease & $\left(r=.278^{*}, p=.022\right)$ \\
Neurologic & $(r=.085, p=.489)$ \\
Hematologic Disease & $\left(r=-.264^{*}, p=.030\right)$ \\
Morbidity & $\left(r=.554^{*} p=0.000\right)$ \\
Mortality & $(r=.181 p=.139)$ \\
\hline
\end{tabular}

Cardiovascular disease association with renal disease and hematologic disease is significant. Cardiovascular-disease associated mortality has not established, one of the main reasons is the poor performance of tools on assessing the patients with SLE. ${ }^{7}$ In conclusion cardiovascular manifestation is more frequent symptoms with systemic lupus erythematosus (SLE) correlation with disease activity and morbidity but not with mortality. Recommendations: focus research to evaluation and an adequate assessment of cardiovascular complications on the morbidity and mortality of the patients with SLE are still required.

\section{Acknowledgments}

We are grateful to all patients who participated and help in accomplishing this work.

\section{Conflicts of interest}

None.

\section{References}

1. Petri M. Cardiovascular Systemic Lupus Erythematosus, Systemic Lupus Erythematosus. In: Robert G Lahita (Eds.), Elsevier, Netherlands, 2004;pp.913-941.

2. Busteed S, Sparrow P, Molloy C, et al. Myocarditis as a prognostic indicator in systemic lupus erythematosus. Postgrad Med J. 2004;80(944):366-367.
3. Esdaile JM, Abrahamowicz M, Grodzicky T, et al. Traditional Framingham risk factors fail to fully account for accelerated atherosclerosis in systemic lupus erythematosus. Arthritis Rheum. 2001;44(10):2331-2337.

4. Doria A, Iaccarino L, Sarzi-Puttini P, et al. Cardiac involvement in systemic lupus erythematosus. Lupus. 2005;14:683.

5. Rho YH, Chung CP, Oeser A, et al. Novel cardiovascular risk factors in premature coronary atherosclerosis associated with systemic lupus erythematosus. J Rheumatol. 2008;35(9):1789-1794.

6. Lalani TA, Kanne JP, Hatfield G, et al. A Imaging Finding in Systemic Lupus Erythematosus. Radiographics. 2004;24(4):1069-1086.

7. Nikpour M, Urowitz MB, Ibanez D, et al. Relationship between cardiac symptoms, myocardial perfusion defects and coronary angiography findings in systemic lupus erythematosus. Lupus. 2011;20(3):299-304.

8. Singh JA, Woodard PK, Roman VGD, et al. Cardiac magnetic resonance imaging abnormalities in systemic lupus erythematosus: a preliminary report. Lupus. 2005;14(2):137-144.

9. Fairfax MJ, Osborn TG, Williams GA, et al. Endomyocardial biopsy in patients with systemic lupus erythematosus. J Rheumatol. 1988;15(4):593-596. 